\title{
Cluster size distribution of infection in a system of mobile agents
}

\author{
M.C. González ${ }^{a, *} \underline{H}_{\text {. J. Herrmann }}^{a, b}$ A. D. Araújo ${ }^{a, b}$ \\ ${ }^{a}$ Institute for Computational Physics, University of Stuttgart, Pfaffenwaldring 27, \\ 70569 Stuttgart, Germany \\ ${ }^{\mathrm{b}}$ Departamento de Fúsica, Universidade Federal do Ceará, 60451-970 Fortaleza, \\ Brazil
}

\begin{abstract}
Clusters of infected individuals are defined on data from health laboratories, but this quantity has not been defined and characterized by epidemy models on statistical physics. For a system of mobile agents we simulate a model of infection without immunization and show that all the moments of the cluster size distribution at the critical rate of infection are characterized by only one exponent, which is the same exponent that determines the behavior of the total number of infected agents. No giant cluster survives independent on the magnitude of the rate of infection.
\end{abstract}

Key words: Non-equilibrium phase transitions, Contact process, Number of Clusters, Epidemic Dynamics

PACS: 89.75.-k, 87.23.Ge, 64.60.H

\section{Introduction}

In a small and highly urbanized nation like Singapore dengue outbreaks or epidemics are identified as "clusters". A dengue cluster or focus of transmission is defined as at least two confirmed cases, with no recent travel history, that are located within $200 \mathrm{~m}$ of each other (taken as the flight range of the Aedes aegypti) and whose dates of the onset of symptoms are within three weeks of each other [1]. Some efforts have been directed towards the characterization of 'SIS' models of infections, or epidemics without immunization $[2,3,4]$, that

\footnotetext{
* Tel.: +49-711-685-3594; fax: +49-711-6853658.

Email address: marta@ica1.uni-stuttgart.de (M.C. González).
} 
is the state of the particles are healthy or infected, and are susceptible to reinfection after healing, thus the name of the model (SIS: susceptible-infectedsusceptible). Analytical and numerical expressions describe the dynamics of the $S I S$ model in terms of the rate of spreading $\lambda$, the evolution of the survival probability of infection $P(t)$, the mean number of infected agents $n(t)$ and the mean square distance of spreading $R^{2}(t)$ in time, which are quantities difficult to compare with real data of epidemics. This work suggests an application of potential comparison with public health data, analyzing a scaling function for clusters numbers on a SIS model of infection.

The second important ingredient of this work is the mobility of agents, contrasted to most of the models of epidemy where the population is modeled by static networks $[2,3,4]$. In a previous work [5] we showed that the $S I S$ model of infection on a system of mobile agents has critical exponents which depends on the density of the system, i.e spatial correlations and mobility of the agents play an important role. We obtained a crossover from mean field behavior for low densities to static $2 D$-lattices for higher densities. Here we use our model of mobile agents to define clusters of infections and analyze its dependency on the rate of infection $\lambda$ (defined in detail bellow) and on the mobility of the agents.

We propose a time-evolving network model: A link between two moving agents is created when they collide with each other and there is transmission of the infection among them (i.e through infected-susceptible interactions), the link lasts a characteristic time of infection.

We find that the network of clusters of infections remains disconnected and no matter how large the rate of infection, no giant cluster is formed. We show that in the transition to spreading, the moments of the cluster size distribution are described by an exponent $\beta$, which is the exponent that characterizes the fraction of infected mass $F_{I M}=N_{I n f} / N$, defined as the ratio of the number of infected agents $\left(N_{\text {Inf }}\right)$ and the total amount of population $(N)$. Thus the number of clusters depends on $\lambda$, and mobility and spatial correlation of the agents influence its dependency.

\section{Model}

$N$ soft disks, with radius $r_{0}=1$, represent agents which move in a two dimensional cell of linear size $L$, with density $\rho=N / L^{2}$. The system has periodic boundary conditions and is initialized as follows: the agents are placed in the cell with the same velocity modulus $v$ and randomly distributed directions, positions and states: 'infected' or 'susceptible'. If a susceptible agent $i$ collides with an infected agent $j$ (i.e $\left|\boldsymbol{r}_{i}-\boldsymbol{r} j\right|<=2 r_{0}$ ), then $i$ becomes infected. Each infected agent heals and becomes susceptible again after a fixed number of time steps, called the 'time of infection' $\left(\Delta t_{\text {inf }}\right)$, which is a free parameter of 
the model.

The physical interaction of the agents is modeled by molecular dynamic with a leap-frog integration method [7], the interaction potential is a $12-6$ LennardJones truncated potential (see more details in [5]).

The resulting model is a contact process [6], where the infected species become extinct unless the infection spreads rapidly enough. The transition between survival and extinction depends on a critical rate of spreading $\lambda_{c}$ that marks the transition into an absorbing state. The infection rate $\lambda$ is defined as the number of agents one agent infects before healing. For this system,

$$
\lambda \equiv \Delta t_{i n f} / \tau_{f}
$$

where $\tau_{f}$ is the characteristic time of flight between two collisions, which is determined by the density $(\rho)$ and the mean velocity of agents $(\langle v\rangle)$. The critical exponents of the transition to spreading were presented by us for the same kind of system [5], where the study was done in terms of the fraction of infected individuals $\left(F_{I M}\right)$. Here we go further and characterize the behavior of the clusters of infected individuals. When agent $j$ infects agent $i$ a link is created among them, the link lasts until one of them heals, meanwhile each of them continues making links with other susceptible agents through the same rule. A cluster is thus defined as a group of infected agents connected by links. Note that in contrast to percolation, where clusters are given by occupied lattice sites connected by nearest-neighbor distances, for this model each cluster gives a group of agents infected in a given period of time linked by a relation of contagion. Isolated infected agents are regarded as clusters of size unity and any cluster consisting of $s$ connected agents is an $s$-cluster. We borrow the notation from Stauffer's book on percolation theory [8] and define here $n_{s}=N_{s} / N$ as the number of $s$-clusters per agent, where $N_{s}$ is the number of clusters of size $s$ and $N$ the total number of agents in the system. For different values of $\lambda$, in the next section we present the results of the calculation of the first three moments of the cluster size distribution. Namely: $\sum_{s} n_{s}$, $\sum_{s} s n_{s}, \sum_{s} s^{2} n_{s}$. Those quantities give us, respectively, information about: the total number of clusters, the fraction of infected agents and the mean size of clusters. In order to keep the analogy with the calculation on percolation, we sum over all values of $s$ excluding the largest cluster $\left(S_{\text {major }}\right)$. We also present, the calculations of $F_{\text {major }}=S_{\text {major }} / N$, the fraction of agents that belong to the largest cluster and $F_{I M}=N_{i n f} / N$, the fraction of agents that are infected.

\section{Results}

For a fixed density, we vary $\lambda$ (Eq. 1) changing the time of infection $\left(\Delta t_{\text {inf }}\right)$. Starting with half of the population infected, for rate of infections near $\lambda_{c}$, a 

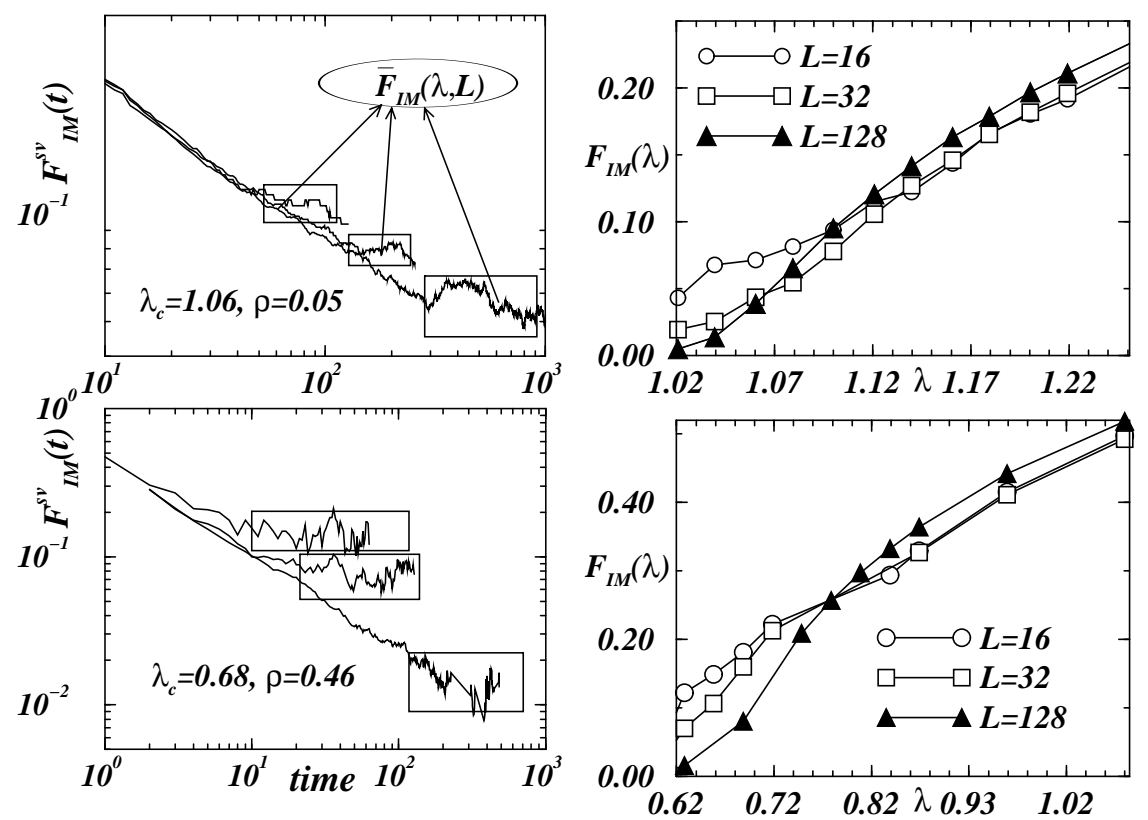

Fig. 1. Left: Fraction of infected individuals from surviving trials versus time at $\lambda=\lambda_{c}$, starting with half of the population infected. At the top, the results for $\rho=0.05$ and $\lambda=1.06$ and at the bottom $\rho=0.46$ and $\lambda=0.68$. System sizes $N=32 \times 32,64 \times 64,128 \times 128$ (from top to bottom). Right: Quasi-stationary fraction of infected agents versus $\lambda$ for the same densities (Top: $\rho=0.05$. Bottom: $\rho=0.46)$.

given trial may end into the absorbing state after a few time steps or it may survive fluctuating with a quasi-stationary fraction of infected agents, marked with windows in the left-side of Fig 1 . The calculations are made averaging on time at the quasi-stationary state, which is described by the surviving trials following an initial transient. The number of time steps of this transient depends on $\lambda$ and on the system size $L$ (see left side of Fig. 1). The data here illustrate how the mean fraction of infected agents $F_{I M}^{s v}(t)$ (the superscript denotes an average restricted to surviving trials) approaches its stationary value $\bar{F}_{I M}(\lambda, N)$ (in the following, we write $\bar{F}_{I M}(\lambda, N)$ just like $\left.F_{I M}(\lambda)\right)$. In the right side of the same figure we see the graph of $F_{I M}(\lambda)$, which becomes sharper increasing the system size. We analyze in detail the number of clusters for the two density values $\rho=0.05$ and $\rho=0.46$, which have critical rate of spreading $\lambda_{c}=1.06$ and $\lambda_{c}=0.68$ respectively. Note that at the critical density $\lambda_{c}$, surviving trials tend to stationary values only in the limit $L \rightarrow \infty$. The left side of Fig. 2 is only for pedagogical reasons, in order to illustrate how the number of clusters looks in the quasi-stationary state, we see snapshots of the clusters of infections for different systems densities and the same rate of infection $\lambda=1.5$, here $N=10 \times 10$.

For $\rho=0.05$ and $\lambda \in[1,200]$, the right side of Fig. 2 shows the variation of $F_{I M}(\lambda)$ and $N=32 \times 32$ averaged over 20 different realizations. The insets show the change in time of $F_{\text {major }}$ and $\sum_{s} n_{s}$, for only one realization with $\lambda=$ 


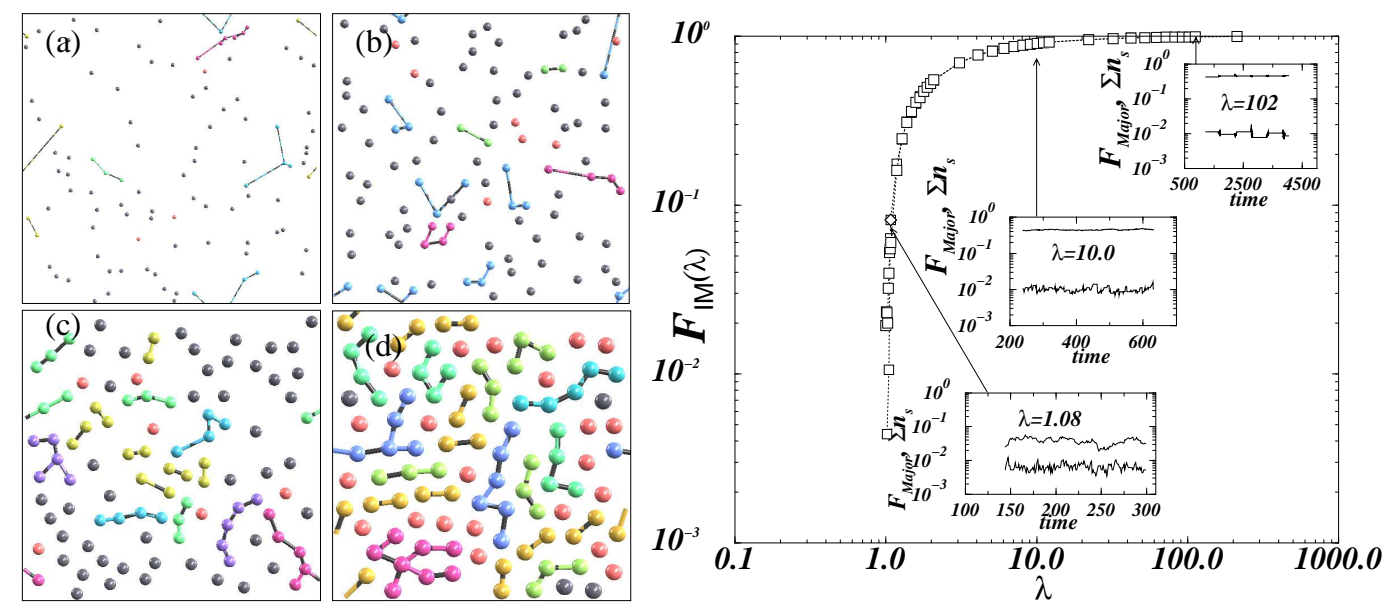

Fig. 2. Left: Snapshots of cluster sizes of infected agents for systems with different densities: (a) $\rho=0.05$, (b) $\rho=0.20$, (c) $\rho=0.40$ and (d) $\rho=0.80$, in all cases $\lambda=1.5$. Right: Quasi-stationary fraction of infected agents varying $\lambda$ over three orders of magnitude (Average over 20 realizations for $\rho=0.05$ and $N=32 \times 32$ ). The insets show the fraction of infected agents in the largest cluster (lower value) and the the first moment of the cluster size distribution (upper value) vs. time, at $\lambda=1.08$, $\lambda=10.0$ and $\lambda=108.0$.

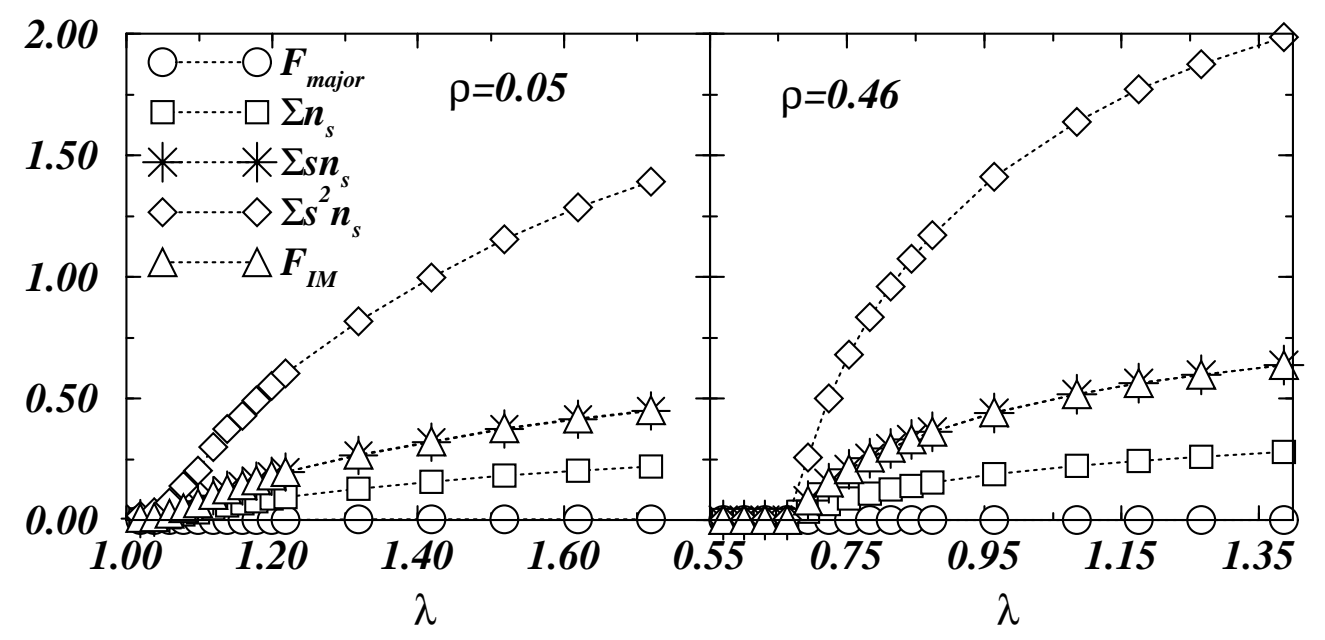

Fig. 3. First three moments of the cluster size distribution, fraction of agents in the largest cluster $\left(F_{\text {major }}\right)$ and fraction of infected agents $\left(F_{I M}\right)$ vs. $\lambda$. Average over 50 trials, system size $N=64 \times 64$. Left: $\rho=0.05$. Right: $\rho=0.46$

1.08, $\lambda=10.0$ and $\lambda=108$. In contrast to percolation results, in this model there is no significant variation of $F_{\text {major }}$ with $\lambda$, and the relation $F_{\text {major }} \ll$ $F_{I M}$ remains. Moreover, the number of clusters $\sum_{s} n_{s}$ grows considerably only near $\lambda_{c}$.

In Fig. 3 for $\rho=0.05$ and $\rho=0.46$, we plot the behavior of the cluster numbers near their respective $\lambda_{c}$. As the largest cluster remains small compared to the 

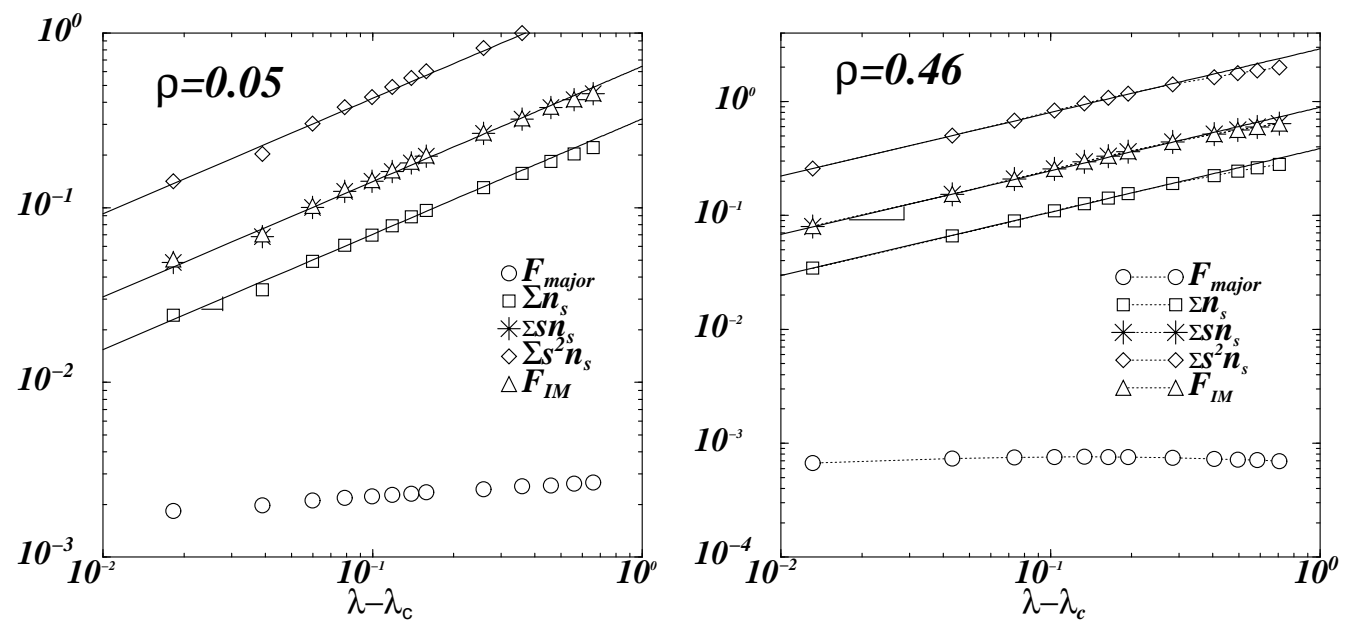

Fig. 4. Same results of Fig. 3 plotted vs. $\left(\lambda-\lambda_{c}\right)$. The solid lines are regressions of the form $m_{i}\left(\lambda-\lambda_{c}\right)^{\beta}$ with $m_{i}$ the coefficient of the $i$ th moment. Left: $\lambda_{c}=1.06$, $\beta=0.66, m_{0}=0.321, m_{1}=2 m_{0}$, and $m_{2}=6 m_{0}$. Right: $\lambda_{c}=0.68, \beta=0.56$, $m_{0}=0.386, m_{1}=2.3 m_{0}$, and $m_{2}=7.5 m_{0}$

total number of agents $\left(S_{\text {major }} \ll N\right)$, we have $F_{I M}(\lambda) \sim \sum_{s} s n_{s}$. Additionally one can see that $\sum_{s} s n_{s}$ and $\sum_{s} s^{2} n_{s}$ show the same critical behavior as $F_{I M}(\lambda)$, plotted in detail in Fig. 4. We observe that all the moments of the cluster size distribution present exactly the same critical behavior than $F_{I M}$, namely $\left(\lambda-\lambda_{c}\right)^{\beta}$, where $\beta$ depends on the density of the system.

\section{Conclusions}

This work showed that the cluster size distribution of infected individuals is described in terms of the spreading rate $(\lambda)$ and the same exponents $(\beta)$ previously known for the total mass of infection. Although the agents are free to move there is a homogeneous size distribution of infected clusters at the critical rate of infection, and we did not find any critical exponent associated with the cluster sizes. Comparing with the traditional SIS model on a static network we confirm that mobility and spatial correlations change the value of the critical exponent $\beta$ of the fraction of infected population, and to the same extent the cluster size distribution of infection.

\section{References}

[1] B. Loh and R. Jn Song, Dengue Bulletin, 25,74-78,(2001).

[2] D. Mollison, J.R. Statist. Soc. B, 39, p. 283 (1977).

[3] P. Grassberger, J. Phys. A: Math. Gen., 22, p. 3673 (1989). 
[4] R. Pastor-Satorras and A. Vespignani, Phys. Rev. E, 63, 066117, (2001).

[5] M. C. González, H. J. Herrmann, Physica A, 340, p.741 (2004).

[6] J. Marro and R. Dickman, Nonequilibrium phase transitions in lattices models (Cambridge University Press, Cambridge , 1999).

[7] D. C. Rapaport, The Art of molecular dynamics simulation (Cambridge University Press, Cambridge, 1995).

[8] D. Stauffer, Introduction to Percolation Theory (Taylor \& Francis, London, 1985). 